\title{
Reducing Run-time Execution in Query Optimization
}

\author{
Rashmi Singh \\ Galgotias College of \\ Engineering and \\ Technology \\ Greater Noida
}

\author{
Somesh Sharma \\ Galgotias College of \\ Engineering and \\ Technology \\ Greater Noida
}

\author{
Saurabh Singh \\ Galgotias College of \\ Engineering and \\ Technology \\ Greater Noida
}

\author{
Bhawna Singh \\ Galgotias College of \\ Engineering and \\ Technology \\ Greater Noida
}

\begin{abstract}
The main objective of query processor is to generate the most efficient query results. Using an apt execution plan, query minimizes cost of execution for results. The order of accessing a source table is very important during query execution. The best execution plan from possible ones is presented by Query optimizer. The paper discusses various stages of query optimization using execution plan. It gives the analysis of indexes, type of expressions \& joins used in the execution plan of the query. The approach gets the estimate of the cost of query joins in a query at compile time. These estimates help in the construction of a query plan at compile time and then executed at run-time.
\end{abstract}

\section{Keywords}

Execution plan,query optimization,compile time,run time,joins.

\section{INTRODUCTION}

In today's object oriented programming languages, need of optimization of query is increasing. Introduction of various processing methods and constructs are being analysed. These new methods have also shown great results in increasing the comprehensibility of programs and the ability of programmers. In SQL, different execution plans are present to optimize the queries. In other programming languages, the constructs allows queries to be written concisely and concretely. The advantages of using query constructs in place of typical representation are well justified. The queries written using other APIs are less clear and concrete than explicit queries. This method provides an avenue for developers to be more productive and work at a higher level of abstraction.

In query processing, there are well described as stages. Firstly, the query processor accepts SQL syntax, secondly selects a plan for executing the syntax, and then executes the chosen plan. Following diagram shows functioning of query processor [1][2][5].

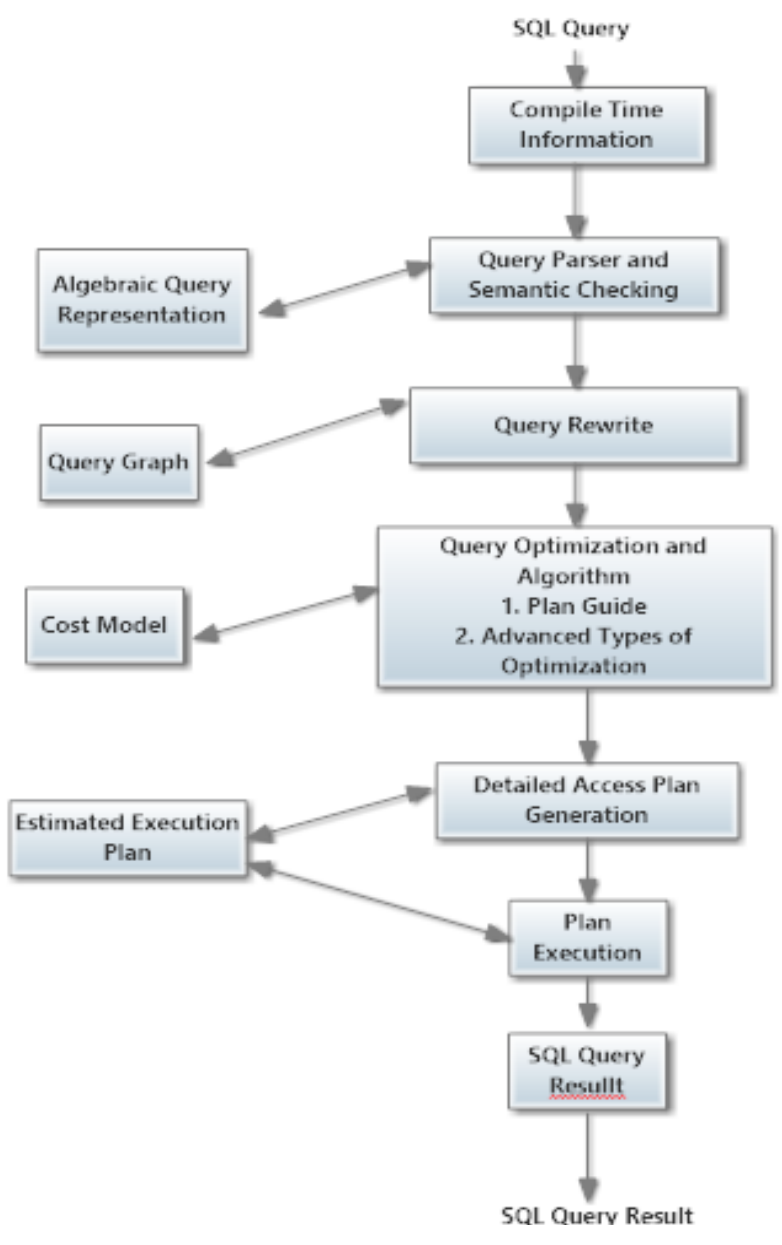

Fig 1: Functioning of query processor.

\subsection{Parsing and Semantic Checking}

For processing SQL query, the database server employs parser to parse the query to perform syntactic and semantic checking of the query. It is done by transforming the query into a parse tree which is the algebraic representation of the query [6] [7] [8].

\subsection{Query Rewrite}

This step involves only complex query which involves joins, predicates, grouping, etc. In this phase query is presented in the form of an annotated parse tree. Query experiences iterative transformations in accordance to the heuristics used. After that, other steps like joining elimination, predicating normalization, selecting operation are performed and at last, project operation and many transformation rules are performed [9].

\subsection{Query Pre-optimization Phase}


This stage involves pre-optimization phase and enumeration phase. In pre-optimization phase, all predicates, indexes, joins used in the access plan are studied by query analyse. Furthermore, include designing alternative plans for the query. In Enumeration phase, various possibilities of the access plans for the query are collected. The most favourable access plan is chosen utilizing join algorithm, cost estimation method, access method, and selectivity.

\subsubsection{Join Selection}

Join Selection is third in a series of steps for query optimization. In case of the query that is multi-table query or a self- join, the query optimizer analyses join selection and selects low cost join strategy. It evaluates the cost by using a number of factors, expected number of reads and the amount of memory required. The choice can be made between three basic strategies for processing joins: nested loop joins, merge joins, and hash joins.

\section{- Nested Loop Joins}

Nested Loop Joins are mainly for smaller tables. Method of Iteratively scanning the rows of one table and matching with a second table with the help of index is called nested loop join. Hash join is used in absence of nested join. There are 3 types of nested Join. The search scanning an entire table or index is called a naive nested loop join. The search using an index, called an index nested loops join. The index is made as part of the query plan, called a temporary index nested loops join. In temporary index nested loop join, the index is destroyed after execution of the query.

\section{- Merge Joins}

Being more efficient for large tables with the key columns sorted, merge join is used in sorting of two inputs on the join column. In case of the inputs are already sorted then less $\mathrm{I} / \mathrm{O}$ is required to process when the join used is one to many. Many merge join make use of a temporary table to store rows instead of discarding them. One of the inputs must rewind to start of the duplicates when duplicate values from each input. It is a fast joining method, but it can be expensive if sort operation is required

\section{- Hash Joins}

Hashing matched a particular data item with an already present value by division of the existing data into groups on the basis of similar property. Hash Bucket has the data with the same value. Hash bucket checks with existing data to find a match. The smaller input is taken as build input during hash joining. The storage in buckets, also known as hash tables, takes place in the form of linked lists, in which each entry contains only columns from building input that are needed. Hash joins are comparatively effective in set- matching operations such as intersection, union, semi-join, full outer join. The set of columns in the equality predicate is called hash key which contributes in the hash function. We have used Adventure Works database. From database we have used Sales.Customer and Sales.SalesOrderHeader tables for demonstrating hash join.

\subsubsection{Index Selection}

Association of table with an index improves the retrieval speed of rows from table [9-11]. There are two types of indexes: Clustered index and Non-Clustered index. Clustered Indexes simply sort and stores the data rows in the table centred on the column selected as the key. When there is a clustered index in the table, the table is known as clustered table. A table with no clustered index is called as a heap suggesting an unordered structure. Consider the table customer and the following query which creates an index on Cust_id of Customer table.

Non-clustered index comprises the non-clustered index key values and each key value entry has a pointer to the data row that contains the key value. The following query creates nonclustered index on Cust_Category of Customer table.

The non-clustered column has depended on the Clustered column in the database. The Cust_category column with distinct values will store the clustered index columns values along with it.

\subsubsection{Plan Selection}

It is the fourth step in query optimization. It is decided by the cost of a given plan, in terms of required CPU preprocessing and $\mathrm{I} / \mathrm{O}$, and query execution time. Hence it is known as Cost- Based plan.

The optimizer's work involves the generation and evaluation of many plans and choosing the lowest cost plan. It solely depended on the plan as it will execute the query relatively fast, using the least amount of resources, CPU and I/O. The optimizer may take a less efficient plan in case of it will take more time to evaluate many plans in comparison to running a less efficient plan. For example, a simple query having a single table with no indexes and no aggregates or calculations is present in these the query, then the optimizer will use single, trivial plan for these types of queries to save time that will wasted in choosing an optimal plan. Otherwise, the optimizer will do cost based calculation to plan selection in case of non-trivial query. For carrying out this, optimizer depends upon on statistics of the execution plan [11].

\subsection{Detailed Plan Execution}

This phase takes the top plan and made the graphical view of the query. Graphical view is available in Interactive SQL. Graphical View has a tree like structure where each node is a physical operator implementing specific relational algebra operation.

\subsection{Plan Execution}

Best execution plans built in previous phase used in the computation of the result of the query.

The input to the optimizer involves the query, the database schema (table and index definitions), and the database statistics. The execution plan is the output of the query optimizer. Query optimizer based on the following components determines the execution plan of the optimizer.

- Access Method: decides how to access the data. It can be Table Scan or Index Scan etc.

- Join Method: Decides how to join tables to each other. It can be a hash join or merge join etc.

- Order of Join: decides the sequence of joining table.

- Cardinality: Depending upon the number of rows, selection of the access method, or join algorithm 
- Join Type: Execution plan also reviews the join type such anti-join, semi-join etc.

- Partition Pruning: Query optimizer checks the partitions required for obtaining the query result.

- Parallel Execution: Query Optimizer checks each operation the plan which is conducted in parallel or not, the data redistribution method used is right or not.

During dealing with query optimization, testing the execution plan for different execution strategies is a must. The selection of the best execution plan includes various factors such as selection of join algorithm, the use of indexes, the order of executing relational algebra operators etc.

\section{QUERY OPTIMIZER ARCHITECTURE}

In this part, a brief outlook on query optimization process in a DBMS has been provided. The execution plans can be decided for a database and a query, which can be used to answer the query. Analyses of all the plans need to be made for selecting the one with best estimated performance. This architecture can allow one to make optimizer, in real systems. Query optimization process has two stages: rewriting and planning. There is only one module in the first stage, the Rewrite and remaining modules are in the second stage.

\subsection{Module Functionality}

\subsubsection{Rewriter}

In this module, given query gets transformed and resulting in equivalent queries that can be more efficient, e.g., replacing views with their respective definition, attuning out of nested queries, etc. The Rewriter transforms query, taking account of the declarative, i.e., static, characteristics of queries without amounting for the actual query costs for the specific DBMS and database concerned. The original query is not processed given the rewriting is known or assumed to always be beneficial; or else, it is forwarded to the next stage. This stage functions at the declarative level of understanding nature of the rewriting transformations.

\subsubsection{Planner}

Being a main module of the ordering stage, it chooses the best execution plan, to be used to generate the answer of the original query, by analysing all other plans which are generated in the previous stage. It utilizes a search strategy; examining the space of execution plans in a descriptive way. The Algebraic Space and the Method-Structure Space are two other modules to decide the space. Additionally, these two modules and the search strategy also optimize the cost, i.e., running time. The Planner compares various execution plans on the basis of their estimated cost so that the cheapest may be chosen. These costs are decided upon by the last two modules of the optimizer, the Cost Model and the Size- Distribution Estimator.

\subsubsection{Algebraic Space}

This module decides execution action orders that are to be considered by the Planner. The actions present same result, differing on one parameter, i.e. performance and are represented in relational algebra as formulas or in tree form. Given the involvement of the algorithmic side of the objects produced in module and eventually forwarded to the Planner, the whole planning stage is seen as operating at the procedural level.

\subsubsection{Method Structure Space}

This module defines the choices made to implement the processing of each ordered series of actions decided by the Algebraic Space. This choice is made in accordance with the present join methods for each join (e.g., nested loops, merge scan, and hash join), if supported data structures are built on the $y$, if/when duplicates are eliminated, and other implementation characteristics of this sort, which are predetermined by the DBMS implementation. This choice is decided by available indices for involving each relation, which is controlled by the physical representation of each database present in its catalogues. This module generates complete execution plans using an algebraic formula or tree from the Algebraic Space, which specify the implementation of each algebraic operator and the use of any indices.

\subsubsection{Cost Model}

This module presents the arithmetic formulas that are deployed for cost - estimation of execution plans. Each different join method, each different index type access, and in general each distinct kind of step have a cost finding formula. Most of these formulas are simply approximations of what the system actually does and are based on certain assumptions regarding issues like buffer management, disk-CPU overlap, sequential vs. random I/O, etc. The essential input parameters to a formula are the size of the buffer pool used by the corresponding step, the sizes of relations or indices accessed, and possibly various distributions of values in these relations. First one is decided by the DBMS for each query; the other two are estimated by the Size-Distribution Estimator.

\subsubsection{Size Distribution Estimator}

This module determines the sizes (and possibly frequency distributions of attribute values) of database relations and estimation of indices as well as (sub) query results. The Cost Model needs these estimates. The module adopts an estimation approach to determine the form of statistics for maintaining catalogs of each database, if any.

\subsection{Plan Guide}

Plan guides are used for performance optimization of queries when we may not to want to change the text of the query directly. Plan guides decide optimization of queries by attaching query hints or a fixed query plan to them. Plan guides can be created to match queries that are executed in the following contexts:

An OBJECT plan guide matches queries that are executed in accordance of Transact-SQL stored procedures, user-defined scalar functions, multi-statement user-defined table-valued functions, and DML triggers.

An SQL plan guide matches queries executed in the context of stand-alone Transact-SQL statements and batches that are not part of a database object. SQL-based plan guides can also be used to match queries that parameterize to a specified form.

A TEMPLATE plan guide matches stand-alone queries that parameterize to a specified form. These plan guides are used to override the current PARAMETERIZATION database SET option of a database for a class of queries.

Plan guide uses any combination of valid query hints. The OPTION clause given in the hints clause is added to the query before it compiles and optimizes during the matching of query in 
plan guide. In a few cases, a query that was matched to a plan guide already has an OPTION clause; the plan guide's query hints replace those in the query. For a plan guide to match a query that already has an OPTION clause, you must include the OPTION clause which specifies the query's text to match in the sp_create_plan_guide statement. If the hints that are specified in the plan guide to be added to the hints that is already present in the query, then instead of replacing them, both the original hints and the additional hints are specified in the OPTION clause of the plan guide.

Some plan guides apply a fixed query plan when aware of an existing execution plan to perform better than the one selected by the optimizer for that query. Taking note of the fact that fixing a plan to a query suggests that adapting the plan by the query optimizer cannot longer change in statistics and index of query. When considering plan guides using fixed query plans, the advantages of applying a fixed plan with the inability to adapt the plan automatically as data distribution and available indexes change are compared.

\section{ADVANCED TYPE OF OPTIMIZATION}

In this section, a brief description of advanced types of optimization is provided that have been given over the past few years [12]. A lot of interesting work has been done on them, e.g., nested query optimization, rule-based query optimization, query optimizer generators, object-oriented query optimization, optimization with materialized views, heterogeneous query optimization, recursive query optimization, aggregate query optimization, optimization with the expensive selection predicates, and query optimizer validation [13].

\subsection{Semantic Query Optimization}

As a form of optimization, Semantic query optimization is mostly related to the Rewriter module.

The core theme deals with usage of integrity constraints present in the database for rewriting a given query into semantically equivalent ones. Further, Planner optimizes them as regular queries and generated the efficient plan to solve the original query. Stating an example, using a hypothetical SQL- like syntax, consider the following integrity constraint:

assert sal-constraint on emp:

sal $>100 \mathrm{~K}$ where job $=$ ISr. Programmer"

Also consider the following query:

select name, oor from emp, dept

where emp.dno $=$ dept.dno and job $=\mid$ Sr. Programmer".

Using the above integrity constraint, the query can be rewritten into a semantically equivalent one to include a selection on sal:

select name, oor from emp, dept

where emp.dno $=$ dept.dno and job $=\backslash$ Sr. Programmer" and sal> $100 \mathrm{~K}$.

The extra selection can assist greatly to find a better plan to answer the query, even during the case of only index in the database being a B+-tree on emp.sal. On the second side, it would make of no use if required index is absent. Owing to all these issues, all proposals for semantic query optimization present and deciding various heuristics or rules making rewritings beneficial and should be applied.
Till now, optimizing individual queries were discussed. Still, many a times during optimization, multiple queries are present simultaneously like queries with unions, queries from multiple concurrent users, queries embedded in a single program, or queries in a deductive system. Optimizing each query alone is a heavy task, one have to devise a global plan to solve this, although possibly suboptimal for each individual query, is ideal for the execution of all of them as a group. Numerous techniques have been mentioned for global query optimization. As a simple example of the problem of global optimization consider the following two queries:

select name, oor from emp, dept

where emp.dno $=$ dept.dno and job $=\backslash$ Sr. Programmer",

select name from emp, dept

where emp.dno $=$ dept.dno and budget $>1 \mathrm{M}$.

Depending on the sizes of the emp and dept relations and the selectivities of the selections, it will be favourable that computation of the entire join once and processing separately the two selections to get the results of the two queries is more efficient than performing the join twice by taking the time of the corresponding selection in consideration. Planner modules are so developed that can analyse all the available global plans and give the optimal one, ultimately it is the objective of global/multiple query optimizers.

\subsection{Dynamic/Parametric Query Optimization}

As mentioned earlier, optimization of embedded takes place once at compile time and are executed multiple times at run time. Owing to the temporal separation between optimization and execution, various parameter's value may be very different during execution from those during the optimization time. This may cause the invalidation of the chosen plan (e.g., if indices used in the plan are no longer available) or simply making it optimal. To resolve this issue, many techniques $[14,15,16]$ have been mentioned that utilize search strategies (e.g., randomized algorithms or the strategy of volcano) to optimize queries along with taking care of all likely values that concerned parameters have during runtime. These techniques deploy usage of the true parameter values at runtime, and then take the optimal plan with little or no overhead. Of a drastically different case, plan switching may take place during query execution through our technique of Rdb/VMS [17], whereby dynamically monitoring how the probability distribution of plan costs changes.

\section{EXPERIMENTAL EVALUATION}

In this section, the performance of the query proposed is evaluated. Several experiments were conducted over certain queries and joins. Following were the observations:

A query can be optimized using HINT query by reducing the cost of performing the join operation. For example,

SELECT OC.Customer, OA.AddressID FROM

SalesLT.Customer AS OC JOIN SalesLT.CustomerAddress AS OA ON OC.CustomerID=OA.CustomerID;

\subsection{Global Query Optimization}




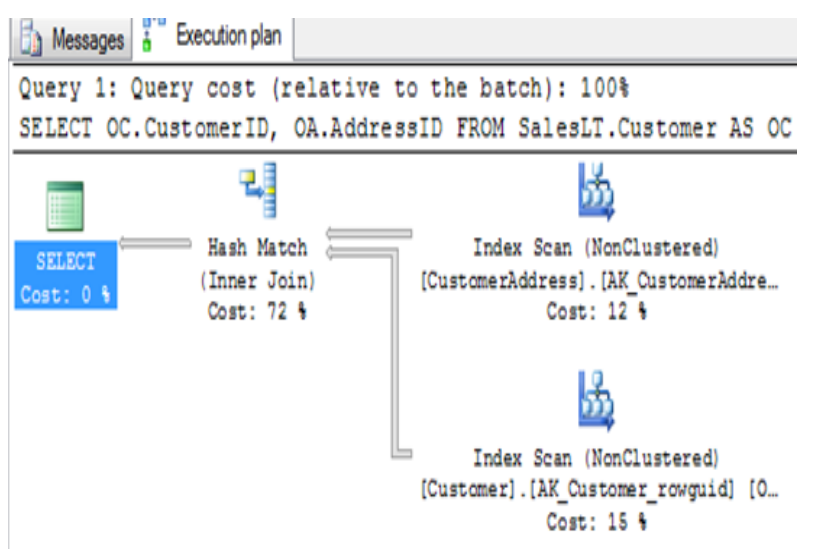

Fig 2: Query cost of join method selected by SQL server

After using HINT query for the join (Merge Join),

SELECT OC.Customer, OA.AddressID FROM

SalesLT.Customer AS OC JOIN SalesLT.CustomerAddress AS

OA ON OC.CustomerID=OA.CustomerD

OPTION (MERGE JOIN);

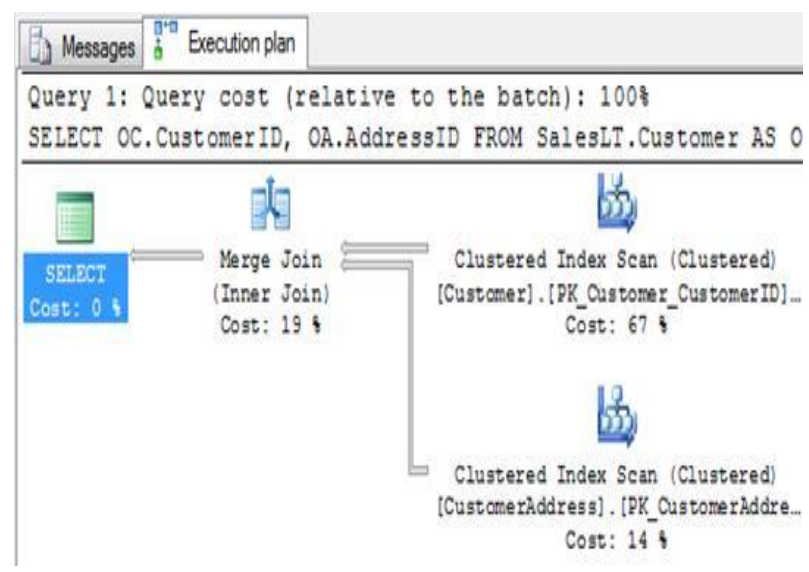

Fig 3: Query cost of merge join using HINT

Now, using HINT queries for implementing Nested Loop Join,

SELECT OC.Customer, OA.AddressID

FROM SalesLT.Customer AS OC JOIN

SalesLT.CustomerAddress AS OA ON

OC.CustomerID=OA.CustomerID OPTION(LOOP JOIN);

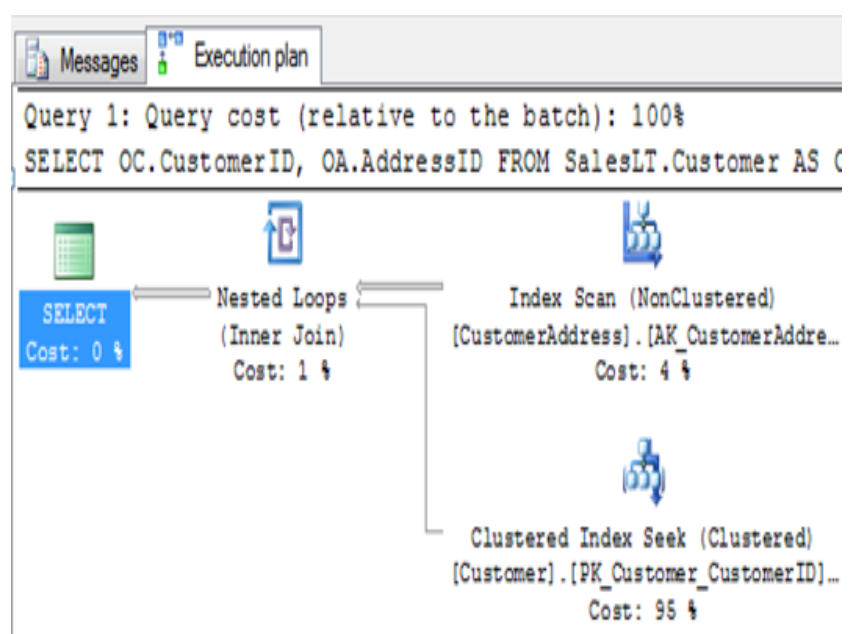

Fig 4: Query cost of nested loop join using HINT

This shows that the cost incurred in using other type of join method using HINT query is reduced, thereby reducing the time taken for execution of the query at runtime.

SQL Server evaluates some constant expressions early to improve query performance, referred to as foldable expressions. This includes

- Arithmetic expressions

- Logical expressions, that contain only constants.

- Built-in functions that are considered foldable by SQL Server. Generally, an intrinsic function is foldable if it is a function of its inputs only and not other contextual information, such as SET options, language settings, database options, and encryption keys.

\section{CONCLUSION}

Given the declarative nature of SQL, there are numerous alternative ways with performance level to execute a given query. When query is submitted to the database, evaluation of different possible plans for executing the query is done by optimizer and generates the best alternative.

In Query Analysis phase, optimizer will find search type of expression and Joins. In Index Selection phase, optimizer chooses the best index to use for each table. In join reordering phase, optimizer computes cost. In plan selection, optimizer will select the best plan suitable for a given query.

So while processing the query, a cost based query optimizer in SQL server gives the effective access path to reduce the total time of execution of the query during runtime.

\section{REFERENCES}

[1] RamezElmasri and Shamkant B. Navathe. Fundamentals of Database Systems, second edition. Addison-Wesley Publishing Company, 1994.

[2] AviSilbershatz, Hank Korth and S. Sudarshan. Database System Concepts,4th Edition. McGraw-Hill, 2002

[3] Henk Ernst Blok, DjoerdHiemstra and Sunil Choenni, Franciska de Jong, Henk M. Blanken and Peter M.G. Apers. Predicting the cost- quality trade-off for information retrieval queries: Facilitatiing database design and query optimization. Proceedings of the tenth international 
conference on Information and knowledge management, October 2001, Pages 207-214.

[4] Reza Sadri, Carlo Zaniolo, Amir Zarkesh and JafarAdibi. Optimization of Sequence Queries in Database Systems. In Proceedings of the twentieth ACM SIGMODSIGACTSIGART symposium on Principles of database systems, May 2001, Pages 71-81.

[5] G. Antoshenkov, "Dynamic Query Optimization in RdblVMS', Proc. IEEE Int '1. Conf on Data Eng., Vienna, Austria, April 1993,538.

[6] C. Mohrm, D. Haderle, Y. Wang, and J. Cheng, "Single Table Access Using Multiple Indexes: Optimization, Execution and Concurrency Control Techniques", Lecture Notes in Comp. Sci. 416 (March 1990), 29, Springer Verlag,

[7] K. Ono and G, M, Lehman, "Measuring the Complexity of Join Enumeration in Query Optimization", Proc. Int '1. Con\$ on Ve Large Data Bases, Brisbane, Australia, August 1990,314 .

[8] G. Graefe and W. J. McKenna, "The Volcano Optimizer Generato Extensibility and Efficient Search", Proc. IEEE Int '1. Con\$ on Data Eng., Vienna, Austria, April 1993,209.

[9] W. Hasan and H. Pirahesh, "Query Rewrite Optimization in Starburst”, Comp. Sci. Res. Rep., SanJose, CA, August 1988.

[10] T.Sellis, "Multiple query optimization", IEEE transactions on knowledge and data Engineering, Vol-2, June-1990

[11] K. Shim, T.Sellis ,D.Nau, "Improvements on algorithms for multiple query optimization " ,IEEE transactions on knowledge and data Engineering, 12, 1994, pp.197-222.

[12] M. M. Astrahan et al. System R: A relational approach to data management. ACM Transactions on DatabaseSystems, 1(2):97\{137, June 1976

[13] P.G.Selinger,M.M.Astrahan,D.D.Chamberlin,R.A.Lorie, and T.G.Price.Access path selection in a relational database management system. In Proc.ACM- SIGMOD Conf. on the Management of Data, pages 23\{34, Boston, MA, June, 1979

[14] G. Graefe and K. Ward. Dynamic query evaluation plans.InProc.ACM-SIGMOD Conference on the Management of Data, pages 358\{366, Portland, OR, May 1989.

[15] Y. Ioannidis, R. Ng, K. Shim, and T. K. Sellis. Parametric query optimization. In Proc.18thInt.VLDBConference, pages $103\{114$, Vancouver, BC, August 1992.

[16] R. Cole and G. Graefe. Optimization of dynamic query evaluation plans. In Proc.ACMSIGMODConferenceontheManagementofData, pages 150\{160, Minneapolis,MN, June 1994. 